\title{
On buried points and phase transition points in the Julia sets concerning renormalization transformation
}

Junyang Gao*

\section{"Correspondence:}

gaojy@cumtb.edu.cn

School of Science, China University

of Mining and Technology, Beijing,

100083, China

\section{Springer}

\begin{abstract}
Considering a family of rational maps $T_{n \lambda}$ concerning renormalization transformation, we give a perfect description of buried points and phase transition points in the Julia set $J\left(T_{n \lambda}\right)$. Furthermore, we prove that $J\left(T_{n \lambda}\right)$ contains an open interval where all points are buried points for some parameters $n$ and $\lambda$, which is according to the problem that Curry and Mayer proposed.
\end{abstract}

MSC: $37 F 10 ; 37 F 45$

Keywords: buried point; phase transition; Julia set; renormalization transformation

\section{Introduction}

It is well known that the Julia set of a rational map is often a fractal. We say that a point in the Julia set is a buried point if it does not lie in the boundary of any Fatou component. If all points in some connected component of the Julia set are buried points, we say this component is called a buried component. Since McMullen [1] gave an example of a rational function with so-called buried points and buried components in its Julia set, much interest has been devoted to investigation of the geometrical and topological properties of buried points and buried components [2-7]. In fact, the existence of buried points and buried components shows that the Julia set has very complex topological properties. In this paper, we investigate the properties of buried points of the family of rational maps $T_{n \lambda}$ concerning renormalization transformation, where

$$
T_{n \lambda}(z)=\left(\frac{z^{2}+\lambda-1}{2 z+\lambda-2}\right)^{n}
$$

with two parameters $n \in \mathbb{N}(n>1)$ and $\lambda \in \mathbb{R}$. This physical model can be derived from the limit distribution of zeroes of the partition function in the famous Yang-Lee theory [8-11]. In fact, some interesting relationships among the phase transitions, the critical exponents, the critical amplitudes and the shape of the Julia sets were found in [12]. After this, many works have been devoted to the Julia sets of the renormalization transformation [13-17].

In order to introduce our results, for any given natural number $n>1$, we define the following constants:

$$
\beta_{n}=2+\max _{-2 \leq t \leq 0} \frac{t^{2 n}-2 t^{n+1}+1}{t-1} .
$$

(O2014 Gao; licensee Springer. This is an Open Access article distributed under the terms of the Creative Commons Attribution License (http://creativecommons.org/licenses/by/2.0), which permits unrestricted use, distribution, and reproduction in any medium, provided the original work is properly cited. 
Figure 1 The Julia set $J\left(T_{5 \lambda}\right)$ for $\lambda=1.824$. The white region in the real axis of this figure is indeed a buried open interval.

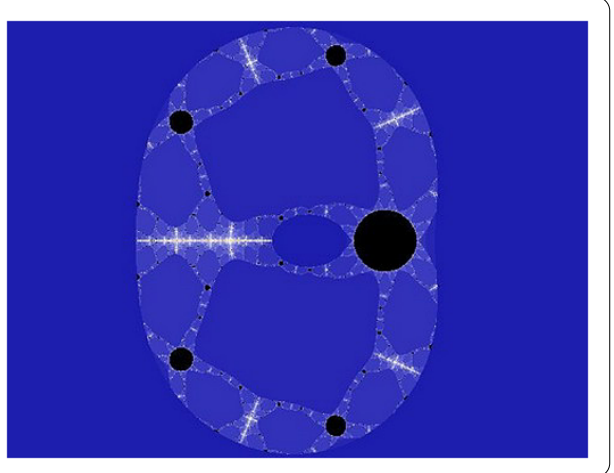

In [14], Qiao proved that $\beta_{n} \in(1,2)$ when $n$ is an even number and $\beta_{n} \in(2,3)$ when $n$ is an odd number. Moreover, he also proved that the equation $(t-1)^{2 n-1}-2(t-1)^{n}-t+3=0$ has one unique real solution $\gamma_{n} \in(2,3]$ when $n$ is an even number. Furthermore, in this paper we prove the following.

Theorem 1 Suppose that $T_{n \lambda}$ is defined as (1), we have

(1) if $n$ is an even number, then $J\left(T_{n \lambda}\right)$ contains buried points if and only if $\lambda \in\left(\beta_{n}, \gamma_{n}\right)$;

(2) if $n=3$, then $J\left(T_{n \lambda}\right)$ contains buried points if and only if $\lambda \in\left[3-\sqrt{2}, \beta_{n}\right]$;

(3) if $n>3$, then $J\left(T_{n \lambda}\right)$ contains buried points if and only if $\lambda \in\left[\lambda_{\alpha}, \beta_{n}\right]$, where $\lambda_{\alpha} \in(1,2)$ is an absolute constant.

In [7], Curry and Mayer proposed some questions about buried points. One of these questions is the following:

Is there the set of buried points whose components are not either points, circles, or the irrational points of the Sierpinski curve?

In this paper, considering the Julia sets $J\left(T_{n \lambda}\right)$, we prove that the set of buried points may contain an open interval of the real axis $\mathbb{R}$. In fact, we have the following.

Theorem 2 Suppose that $n>3$ is an odd number, then there exists $\lambda \in(1,2)$ such that $J\left(T_{n \lambda}\right)$ contains an open interval in which all points are buried points (see Figure 1).

For this physical model, it is well known that there is a specific physical significance about the intersection points between the Julia set $J\left(T_{n \lambda}\right)$ and the positive real axis $\mathbb{R}^{+}$ when $\lambda$ is a positive integer. In fact, these intersection points are the locations of phase transitions of this model. A natural problem is how to distribute on $\mathbb{R}^{+}$the locations of phase transitions? In the following we will show that the number of this kind of intersection points may be $1,2,3$, or $\infty$. Denote by $\#\left(J\left(T_{\lambda}\right) \cap \mathbb{R}^{+}\right)$the number of the intersection points of $J\left(T_{\lambda}\right)$ and $\mathbb{R}^{+}$. Then we have the following.

Theorem 3 If $\lambda$ is a positive integer, then

(1) $\#\left(J\left(T_{n \lambda}\right) \cap \mathbb{R}^{+}\right)=\infty$ for any even number $n \geq 2$ and $\lambda=1$ (see Figure 2 );

(2) $\#\left(J\left(T_{n \lambda}\right) \cap \mathbb{R}^{+}\right)=3$ for any odd number $n \geq 3$ and $\lambda=1$ (see the left figure in Figure 3 );

(3) $\#\left(J\left(T_{n \lambda}\right) \cap \mathbb{R}^{+}\right)=2$ for any positive $n \geq 2$ and $\lambda \in\left[2,2+\frac{1}{\sqrt[n-1]{2}-1}\right.$ ) (see the immediate figure in Figure 3);

(4) $\#\left(J\left(T_{n \lambda}\right) \cap \mathbb{R}^{+}\right)=1$ for any positive $n \geq 2$ and $\lambda \in\left[2+\frac{1}{\sqrt[n-1]{2}-1},+\infty\right)$ (see the right figure in Figure 3). 

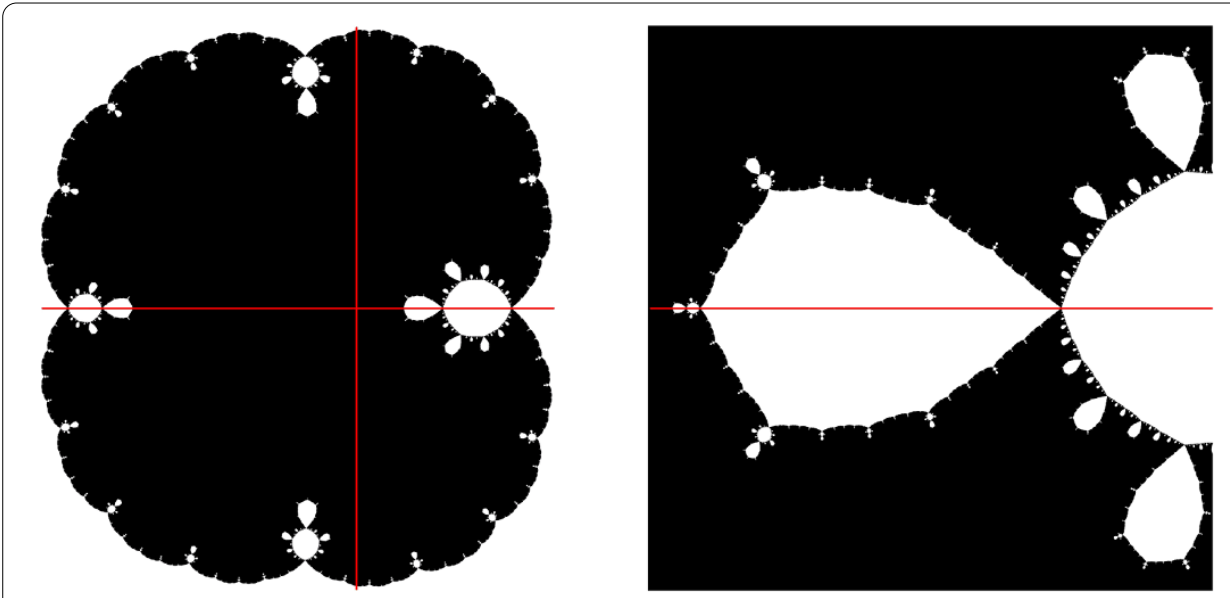

Figure 2 The Julia set of $J\left(T_{41}\right)$ and its enlargement of one location of phase transitions with 192 times.

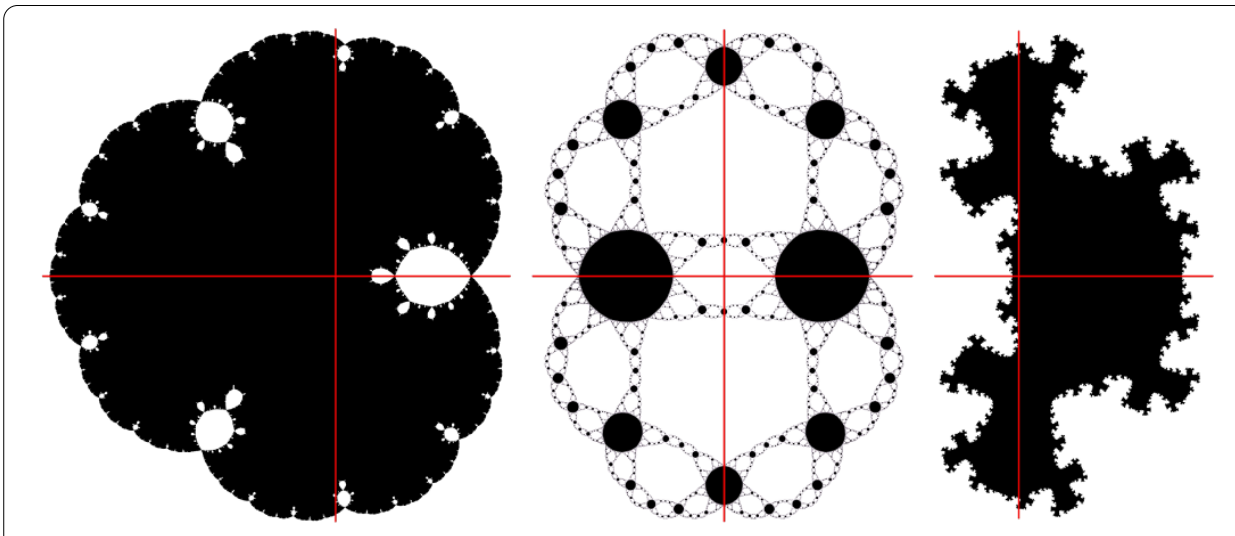

Figure 3 The Julia set of $J\left(T_{31}\right), J\left(T_{42}\right)$ and $J\left(T_{35}\right)$.

\section{Some notations and preliminary results}

Let $f$ be a rational map with degree $d \geq 2$ from a complex sphere $\overline{\mathbb{C}}$ to itself. The notation $f^{k}$ means the $k$ th iteration of $f$. A point $z$ is called a critical point if $f^{\prime}(z)=0$. A point $z$ is called a periodic point if $f^{k}(z)=z$ for some $k \geq 1$, the minimal of such $k$ is called the period of $z$. For a periodic point $z_{0}$ with periodic $k$, the multiplier of $z_{0}$ is defined as $\left(f^{k}\right)^{\prime}\left(z_{0}\right)$. The periodic point $z_{0}$ is either attracting, indifferent, or repelling according to $\left|\left(f^{k}\right)^{\prime}\left(z_{0}\right)\right|<1$, $\left|\left(f^{k}\right)^{\prime}\left(z_{0}\right)\right|=1$, or $\left|\left(f^{k}\right)^{\prime}\left(z_{0}\right)\right|>1$. The Julia set, denoted by $J(f)$, is the closure of repelling periodic points. Its complement is called the Fatou set, denoted by $F(f)$. A component $D$ of $F(f)$ is called completely invariant if $f(D)=f^{-1}(D)=D$. Moreover, if $D$ is a completely invariant component, then $J(f)=\partial D$. Let $P(f)$ be the post-critical set of $f$, i.e., the closure of the forward orbits of critical points. It plays a crucial rule in the study of complex dynamics. For the classical results in complex dynamics, see $[18,19]$ and $[20]$.

In order to prove our theorems, we need the following lemmas.

Lemma $1([5,6])$ Let $f$ be a rational map $f$ with $\operatorname{deg} f \geq 2$ and $J(f)$ connected and locally connected, then $J(f)$ contains buried points if and only if $f^{2}$ has no completely invariant Fatou component. 
Lemma $2([17])$ If $J\left(T_{n \lambda}\right)$ is connected, then it is locally connected.

Lemma 3 ([17]) If $n \geq 3$ is an odd integer, then $T_{n \lambda}$ has only four real fixed points $q_{1}, q_{2}, 1, q_{3}$ $\left(q_{1}<q_{2} \leq 0, q_{3}>1\right)$ for $\lambda \in[1,2)$.

Lemma 4 ([17]) Suppose that $q_{2}$ is the fixed point stated in Lemma 3. If $n=3$ and $\lambda \in(1,2)$, then $q_{2}$ is an attracting fixed point. If $n>3$ is an odd integer, then there exist two numbers $\lambda_{\alpha}$ and $\lambda_{\beta}\left(\lambda_{\alpha}<\lambda_{\beta}\right)$ in $(1,2)$ such that

(1) $q_{2}$ is an attracting fixed point for $\lambda \in\left(1, \lambda_{\alpha}\right) \cup\left(\lambda_{\beta}, 2\right)$;

(2) $q_{2}$ is a parabolic fixed point and $T_{n \lambda}^{\prime}\left(q_{2}\right)=-1$ for $\lambda=\lambda_{\alpha}$ or $\lambda=\lambda_{\beta}$;

(3) $q_{2}$ is a repelling fixed point for $\lambda \in\left(\lambda_{\alpha}, \lambda_{\beta}\right)$.

Lemma 5 ([14] and [15])

(1) If $n \geq 2$ is an even integer, then $T_{n \lambda}^{2}$ contains a completely invariant Fatou component if and only if $\lambda \in\left(-\infty, \beta_{n}\right] \cup\left(\gamma_{n},+\infty\right)$. Furthermore, $J\left(T_{n \lambda}\right)$ is connected for $\lambda \in\left(\beta_{n}, \gamma_{n}\right)$.

(2) If $n>1$ is an odd integer, then $T_{n \lambda}^{2}$ contains a completely invariant Fatou component for $\lambda \in(-\infty, 1] \cup\left(\beta_{n},+\infty\right)$. Furthermore, $T_{n \lambda}^{2}$ contains at most three Fatou periodic (not completely invariant) cycles for $\lambda \in\left[2, \beta_{n}\right]$.

\section{Proof of Theorem 1}

For $\lambda \neq 0$, by (1), we have

$$
T_{n \lambda}^{\prime}(z)=\frac{2 n(z-1)(z+\lambda-1)\left(z^{2}+\lambda-1\right)^{n-1}}{(2 z+\lambda-2)^{n+1}} .
$$

So $T_{n \lambda}$ has only four critical values $1, \infty, 0$ and $T_{n \lambda}(-\lambda+1)=(-\lambda+1)^{n}$.

By Lemma 1, Lemma 2 and Lemma 5 , it is easy to see that $J\left(T_{n \lambda}\right)$ contains buried points if and only if $\lambda \in\left(\beta_{n}, \gamma_{n}\right)$ for even integer $n$. Similarly, if $n>1$ is an odd number, $J\left(T_{n \lambda}\right)$ contains no buried point for $\lambda \in(-\infty, 1] \cup\left(\beta_{n},+\infty\right), J\left(T_{n \lambda}\right)$ contains buried points for $\lambda \in\left[2, \beta_{n}\right]$. Hence we need only to investigate the case that $n>1$ is an odd integer and $\lambda \in(1,2)$.

Obviously, 1 and $\infty$ are two super-attracting fixed points of $T_{n \lambda}$ for $\lambda \in\left(1, \beta_{n}\right]$. In the following we denote by $A_{\lambda}(1)$ and $A_{\lambda}(\infty)$ two Fatou components of $T_{n \lambda}$ that contain 1 and $\infty$, respectively. It is easy to see that $T_{n \lambda}(x)$ is monotone increasing from $q_{1}$ to $-(\lambda-1)^{n}$ on $\left(q_{1}, 1-\lambda\right)$ and monotone decreasing from $-(\lambda-1)^{n}$ to $-\infty$ on $\left(1-\lambda,-\frac{\lambda}{2}+1\right)$, then $q_{2}<T_{n \lambda}(1-\lambda)=-(\lambda-1)^{n}$. It follows that there exists a unique point $q_{1}^{*} \in\left(1-\lambda,-\frac{\lambda}{2}+1\right)$ such that $T_{n \lambda}(x)>q_{1}$ for $x \in\left(q_{1}, q_{1}^{*}\right)$ and $T_{n \lambda}(x)<q_{1}$ for $x \in\left(q_{1}^{*},-\frac{\lambda}{2}+1\right)$. By a similar discussion, there exists a unique point $q_{3}^{*} \in\left(-\frac{\lambda}{2}+1,1\right)$ such that $T_{n \lambda}(x)>q_{3}$ for $x \in\left(-\frac{\lambda}{2}+1, q_{3}^{*}\right)$ and $T_{n \lambda}(x)<q_{1}$ for $x \in\left(q_{1}^{*},-\frac{\lambda}{2}+1\right)$. Hence we get

$$
1-\lambda<q_{2}<(1-\lambda)^{2}<q_{1}^{*}<1-\frac{1}{2} \lambda<q_{3}^{*}<1<q_{3} .
$$

Furthermore,

$$
\left[-\infty, q_{1}\right) \cup\left(q_{3}, \infty\right] \subset A_{\lambda}(\infty), \quad\left(q_{3}^{*}, q_{3}\right) \subset A_{\lambda}(1), \quad\left(q_{1}^{*}, q_{3}^{*}\right) \subset D\left(-\frac{\lambda}{2}+1\right)
$$


and

$$
T_{n \lambda}\left(\left[q_{1}, q_{1}^{*}\right]\right)=\left[q_{1},(1-\lambda)^{n}\right] \subset\left[q_{1}, q_{1}^{*}\right]
$$

where $D\left(-\frac{\lambda}{2}+1\right)$ is the preimage of $A_{\lambda}(\infty)$ containing $-\frac{\lambda}{2}+1$. The fixed points $q_{1}$ and $q_{3}$ are repelled. Below we prove

$$
q_{1}^{*}>0,=0,<0 \quad \Leftrightarrow \quad \lambda \in\left(1,2-\frac{1}{1+\sqrt[n-1]{2}}\right),=2-\frac{1}{1+\sqrt[n-1]{2}}, \in\left(2-\frac{1}{1+\sqrt[n-1]{2}}, 2\right),
$$

respectively.

In fact, set $x=\frac{\lambda-1}{2-\lambda}$, then

$$
T_{n \lambda}^{2}(0)=T_{n \lambda}(0)\left(\frac{x^{2 n-1}+\frac{1}{x+1}}{2 x^{n}+\frac{1}{x+1}}\right)^{n} .
$$

It can be verified that

$$
\frac{x^{2 n-1}+\frac{1}{x+1}}{2 x^{n}+\frac{1}{x+1}}>1,=1, \text { or }<1
$$

is equivalent to $x>\sqrt[n-1]{2},=\sqrt[n-1]{2}$, or $<\sqrt[n-1]{2}$, respectively. We get (5) since $T_{n \lambda}(0)<0$.

(I) If $n=3$ and $\lambda \in\left(1,2-\frac{1}{\sqrt[n-1]{2}+1}\right)$. It is easy to verify that $\left(q_{1}, q_{1}^{*}\right) \subset A_{\lambda}\left(q_{2}\right)$ since $T_{3 \lambda}(x)>x$ for $x \in\left(q_{1}, q_{2}\right)$. Hence $0 \in A_{\lambda}\left(q_{2}\right)$. Next we define

$$
\Gamma(\lambda)=\left\{z:\left|z+\frac{\lambda}{2}-1\right|=\frac{\lambda}{2}\right\} \cap\{z: \operatorname{Re} \mathbf{z} \leq \mathbf{0}\} .
$$

It follows that

$$
T_{3 \lambda}(\Gamma(\lambda))=\left[-(\lambda-1)^{n}, 0\right] \subset\left(q_{1}, q_{1}^{*}\right) \subset A_{\lambda}\left(q_{2}\right)
$$

Note that $1-\lambda \in \Gamma(\lambda) \cap\left(q_{1}, q_{1}^{*}\right)$, then $\Gamma(\lambda) \subset A_{\lambda}\left(q_{2}\right)$. Since $T_{3 \lambda}^{-1}(0)= \pm \sqrt{\lambda-1} i \in \Gamma(\lambda)$, then $A_{\lambda}\left(q_{2}\right)$ is completely invariant. This implies that $J\left(T_{3 \lambda}\right)=\partial A_{\lambda}\left(q_{2}\right)$ and thus $J\left(T_{3 \lambda}\right)$ contains no buried point.

If $n=3$ and $\lambda \in\left[2-\frac{1}{\sqrt[n-1]{2}+1}, 2\right)$. By (3) and (5), it is easy to see that $A_{\lambda}\left(q_{2}\right)$ contains only one critical point $z=1-\lambda$ and $\operatorname{deg}\left(\left.T_{n \lambda}\right|_{A_{\lambda}\left(q_{2}\right)}\right)=2$. Hence $A_{\lambda}\left(q_{2}\right)$ cannot be completely invariant since $\operatorname{deg}\left(T_{n \lambda}\right)=5$. Note that $A_{\lambda}(1)$ and $A_{\lambda}(\infty)$ are also not completely invariant, then $J\left(T_{3 \lambda}\right)$ must contain buried points by Lemma 1 .

(II) If $n \geq 5$. Let $\lambda_{\alpha}$ and $\lambda_{\beta}$ be two numbers stated in Lemma 4. Below we prove

$$
\lambda_{\alpha}<2-\frac{1}{\sqrt[n-1]{2}+1}
$$

and

$$
\lambda_{\beta}>2-\frac{1}{\sqrt[n-1]{2}+1} .
$$


By the proof of Proposition 3 in [17], we know that $q_{2}$ is attracting, parabolic, or repelling according to

$$
(2 n+1) t^{2 n}-2 n t^{2 n-1}-2 n t^{n+1}+2(n-1) t^{n}+1>0,=0, \text { or }<0,
$$

respectively, where $t=\sqrt[n]{x} \in(-1,0)$ and $T_{n \lambda}(x)=x$. Set

$$
P_{n}(t)=(2 n+1) t^{2 n}-2 n t^{2 n-1}-2 n t^{n+1}+2(n-1) t^{n}+1 .
$$

Take $t_{0}^{n}=-\frac{1}{2(n-1)}$, it is easy to see that $-t_{0}-t_{0}^{n-1}>\frac{1}{5}$ for $n \geq 5$. By a calculation, we have

$$
P_{n}\left(t_{0}\right)=\frac{1}{n-1}\left(\frac{2 n+1}{4(n-1)}-n\left(-t_{0}-t_{0}^{n-1}\right)\right)<0 .
$$

Note that $P_{n}(0)=1$, then there exists $\alpha \in\left(t_{0}, 0\right)$ such that $P_{n}(\alpha)=0$. We can easily deduce from $T_{n \lambda}(x)=x$ that

$$
\lambda=\phi(t)=\frac{t^{2 n}-2 t^{n+1}+2 t-1}{t-1} .
$$

By the proof of Proposition 2 in [17], we know that $\phi(t)$ is monotone decreasing from $\phi(-1)=2$ to $\phi(0)=1$ on $(-1,0)$. So we conclude that $\lambda_{\alpha}<\lambda_{t_{0}}$ since $q_{2}$ is a parabolic fixed point of $T_{n \lambda_{\alpha}}$ by Lemma 4 .

Put $t_{*}=-t_{0}$, we have

$$
\begin{aligned}
\lambda_{t_{0}} & =\frac{\left(t_{0}^{n}-1\right)\left(t_{0}^{n}+1-2 t_{0}\right)}{t_{0}-1} \\
& =\frac{(2 n-1)}{2(n-1)}\left(2-\frac{(2 n-1)}{2(n-1)\left(t_{*}+1\right)}\right) \\
& =\frac{3}{2}-\left(\frac{(2 n-1)^{2}}{4(n-1)^{2}\left(t_{*}+1\right)}-\left(\frac{1}{n-1}+\frac{1}{2}\right)\right) .
\end{aligned}
$$

It can be proved that $\varphi(n)=\left(1-\frac{4 n-5}{2\left(n^{2}-1\right)}\right)^{n}(n \geq 5)$ is monotone decreasing and it tends to $\frac{1}{e^{2}}$ when $n \rightarrow \infty$. It is obvious that $t_{*}^{n}=\frac{1}{2(n-1)}<\frac{1}{e^{2}}$ for $n \geq 5$. Hence $t_{*}^{n}<\left(1-\frac{4 n-5}{2\left(n^{2}-1\right)}\right)^{n}$, i.e., $t_{*}<\frac{2 n^{2}-4 n+3}{2\left(n^{2}-1\right)}$. By a calculation, we have

$$
\frac{(2 n-1)^{2}}{4(n-1)^{2}\left(t_{*}+1\right)}-\left(\frac{1}{n-1}+\frac{1}{2}\right)>0 .
$$

So $\lambda_{t_{0}}<\frac{3}{2}$ and thus (7) is obvious.

Below we prove (8).

Take $t_{1}^{n}=-\frac{2}{3}$, it is easy to see that $t_{1} \in(-1,-0.85)$ for $n \geq 5$. Furthermore, we have $P_{n}\left(t_{1}\right)<0$. Note that $P_{n}(-1)=3$, there exists $\beta \in\left(-1, t_{1}\right)$ such that $P_{n}(\beta)=0$. Because $\lambda=\phi(t)=\frac{t^{2 n}-2 t^{n+1}+2 t-1}{t-1}$ is monotone decreasing from $\phi(-1)=2$ to $\phi(0)=1$ on $(-1,0)$, it follows that $\lambda_{\beta}>\lambda_{t_{1}}$. Hence

$$
\lambda_{t_{1}}=\frac{\left(t_{1}^{n}-1\right)\left(t_{1}^{n}+1-2 t_{1}\right)}{t_{1}-1}=\frac{5}{3} \cdot\left(\frac{1}{3}-2 t_{1}\right) \cdot \frac{1}{1-t_{1}}>\frac{5}{3} .
$$

We get (8). 
If $\lambda \in\left(1, \lambda_{\alpha}\right)$. By (5) and (7), we know $q_{1}^{*}>0$. By the same discussion as used in the case $n=3$, we can deduce that $A_{\lambda}\left(q_{2}\right)$ is completely invariant for $\lambda \in\left(1, \lambda_{\alpha}\right)$. This implies that $J\left(T_{n \lambda}\right)=\partial A_{\lambda}\left(q_{2}\right)$ and thus $J\left(T_{n \lambda}\right)$ contains no buried point.

If $\lambda \in\left[\lambda_{\alpha}, \lambda_{\beta}\right]$, it is easy to see that $F\left(T_{n \lambda}\right)$ contains at most another $k$-periodic cycle except for $A_{\lambda}(1)$ and $A_{\lambda}(\infty)$. By (3) and (4), we can deduce that $k \geq 2$ since $q_{2}$ is a repelling fixed point by Lemma 4 (in fact, the periodic Fatou components lie on both sides of $q_{2}$ ). Then $T_{n \lambda}^{2}$ cannot contain a completely invariant Fatou component. This shows that $J\left(T_{n \lambda}\right)$ contains buried points.

If $\lambda \in\left(\lambda_{\beta}, 2\right)$. By (5), $q_{1}^{*}<0$. By (3), $T_{n \lambda}(0) \in A_{\lambda}(\infty)$. It shows that $A_{\lambda}\left(q_{2}\right)$ is not completely invariant since it contains only one critical point $z=1-\lambda$ and $\operatorname{deg}\left(\left.T_{n \lambda}^{k}\right|_{A_{\lambda}\left(q_{2}\right)}\right)=2$, and thus $J\left(T_{n \lambda}\right)$ contains buried points. It completes the proof of Theorem 1.

\section{Proof of Theorem 2}

Note that the post-critical set $P\left(T_{n \lambda}\right) \subset \overline{\mathbb{R}}=\mathbb{R} \cup\{\infty\}$. By [17], the Julia set $J\left(T_{n \lambda}\right)$ is connected for $\lambda \in(1,2)$. By (3) and (4), we can prove that

$$
\partial A_{\lambda}(\infty) \cap \overline{\mathbb{R}}=\left\{q_{1}, q_{3}\right\} \quad \text { and } \quad \partial A_{\lambda}(1) \cap \overline{\mathbb{R}}=\left\{q_{3}^{*}, q_{3}\right\}
$$

In fact, let $\Gamma$ be the circle centered at $1-\frac{1}{2} \lambda$ with radius $\frac{1}{2} \lambda$. Then $\Gamma$ passes through critical points $1,1-\lambda$ and $\pm i \sqrt{\lambda-1}$, and $T_{n \lambda}(\Gamma)=\left[(1-\lambda)^{n}, 1\right] \subset \overline{\operatorname{Int}(\Gamma)}$. We conclude that $A_{\lambda}(\infty) \cap \overline{\operatorname{Int}(\Gamma)}=\emptyset$ and $\partial A_{\lambda}(\infty) \cap[1-\lambda, 1]=\emptyset$. For $x \in\left(q_{1}, 1-\lambda\right)$, there exists a positive integer $k$ such that $T_{n \lambda}^{k}(x) \in\left(1-\lambda,(1-\lambda)^{n}\right]$. This implies $x \notin \partial A_{\lambda}(\infty)$. Hence $\partial A_{\lambda}(\infty) \cap \overline{\mathbb{R}}=$ $\left\{q_{1}, q_{3}\right\}$.

Let $\Omega_{0}$ be the component of $\mathbb{C} \backslash T_{n \lambda}^{-1}([-\infty, 0])$ containing 1 . Then $1-\frac{1}{2} \lambda \in \partial \Omega_{0}$ and $A_{\lambda}(1) \subset \Omega_{0}$. Therefore $\overline{A_{\lambda}(1)} \cap\left[q_{1}, q_{1}^{*}\right]=\emptyset$ and $\partial A_{\lambda}(1) \cap \overline{\mathbb{R}}=\left\{q_{3}^{*}, q_{3}\right\}$.

Obviously, $T_{n \lambda}^{-1}\left(A_{\lambda}(\infty)\right)$ has only two components $A_{\lambda}(\infty)$ and $D\left(-\frac{\lambda}{2}+1\right)$. By $T_{n \lambda}: \mathbb{R} \rightarrow$ $\mathbb{R} \backslash\left((1-\lambda)^{n}, 1\right)$ and $\overline{D\left(-\frac{\lambda}{2}+1\right)} \cap \mathbb{R}=\left[q_{1}^{*}, q_{3}^{*}\right] \subset\left((1-\lambda)^{n}, 1\right)$, we know that $\bar{W} \cap \mathbb{R}=\emptyset$ for every component $W$ of $T_{n \lambda}^{-1}\left(D\left(-\frac{\lambda}{2}+1\right)\right)$. Similarly, by (3) and (4), we can deduce that $\overline{W_{*}} \cap \mathbb{R}=\emptyset$ for every component $W_{*}$ of $T_{n \lambda}^{-1}\left(A_{\lambda}(1)\right)$ except for $A_{\lambda}(1)$. Hence the closures of all pullbacks of $W$ and $W_{*}$ are disjoint with $\mathbb{R}$ since $T_{n \lambda}$ maps $\mathbb{R}$ into $\mathbb{R} \cup\{\infty\}$. By [17], we know that there exist at least two real numbers $\lambda_{1}, \lambda_{2} \in(1,2)$ such that $T_{n \lambda_{1}}$ and $T_{n \lambda_{2}}$ are Feigenbaum-like maps. Obviously, $T_{n \lambda_{1}}$ and $T_{n \lambda_{2}}$ contain only two Fatou periodic domains $A_{\lambda}(1)$ and $A_{\lambda}(\infty)$. By the above analysis and (4), we conclude that $\left(q_{1}, q_{1}^{*}\right)$ lies in the Julia sets $J\left(T_{n \lambda_{1}}\right)$ and $J\left(T_{n \lambda_{2}}\right)$, and each point of this open interval does not belong to the boundaries of any Fatou components. This implies that $\left(q_{1}, q_{1}^{*}\right)$ is a buried interval of $J\left(T_{n \lambda_{1}}\right)$ and $J\left(T_{n \lambda_{2}}\right)$ (in fact, by a calculation, there exists $\lambda_{0} \in(1,2)$ such that $1-\lambda_{0}$ is strictly eventually periodic, then $\left(q_{1}, q_{1}^{*}\right)$ is also a buried interval of $\left.J\left(T_{n \lambda_{0}}\right)\right)$. It completes the proof of Theorem 2.

\section{Proof of Theorem 3}

Obviously, $T_{n 1}(z)=\left(\frac{z^{2}}{2 z-1}\right)^{n}$. It has only four critical points $0, \frac{1}{2}, 1$ and $\infty$. Let $\phi(z)=\frac{1}{z}$, then

$$
S(z)=\phi^{-1} \circ T_{n 1} \circ \phi(z)=z^{n}(2-z)^{n}
$$

is a polynomial. Note that $\phi(\infty)=0$, then the immediate basin $A_{\lambda}(0)$ containing $z=0$ of $T_{n 1}$ is completely invariant. Below we prove (1) and (2) of Theorem 3. 
(1) If $n$ is an even number. We consider the real fixed points of $T_{n 1}$, i.e., the set $\{x \in$ $\left.\mathbb{R} \mid T_{n 1}(x)=\left(\frac{x^{2}}{2 x-1}\right)^{n}=x\right\}$. It is easy to see that $T_{n \lambda}(x)$ has no fixed point on $(-\infty, 0)$ since $T_{n \lambda}(x) \geq 0$ for $x \in \mathbb{R}$. By (2), $T_{n 1}(x)$ is monotone increasing on $\left(0, \frac{1}{2}\right)$ from 0 to $+\infty$. Note that $T_{n 1}\left(\frac{1}{2}\right)=+\infty$ and $z=1$ is an attracting fixed point, then there exists at least one fixed point $\alpha \in\left(0, \frac{1}{2}\right)$ such that $T_{n 1}(x)>x$ for $x \in(0, \alpha)$ and $(0, \alpha) \subset A_{\lambda}(0)$.

Next we claim that $\alpha$ is the unique fixed point in $\left(0, \frac{1}{2}\right)$.

Otherwise, if there exists another fixed point $\gamma$ in $\left(0, \frac{1}{2}\right)$. Without loss of generality, we assume $\alpha<\gamma$, then $T_{n 1}(x)>x$ or $T_{n 1}(x)<x$ on $(\alpha, \gamma)$. Note that $T_{n 1}(x)$ is monotone increasing on $\left(0, \frac{1}{2}\right)$, then one of them $(\alpha$ or $\gamma)$ is an attracting fixed point or a parabolic fixed point. Since $T_{n 1}(0)=0, T_{n 1}\left(\frac{1}{2}\right)=+\infty, T_{n 1}(1)=1$ and $T_{n 1}(\infty)=\infty$, then the immediate basin contains no critical point. But the Sullivan theorem [18] says that each immediate basin contains at least one critical point. It is a contradiction. Hence $\alpha$ is the unique fixed point on $\left(0, \frac{1}{2}\right)$. Similarly, we can deduce that there also exists a unique fixed point $\beta$ in $(1,+\infty)$. Hence $T_{n 1}$ has only four real fixed points $0,1, \alpha, \beta, \alpha$ and $\beta$ are two repelling fixed points.

Furthermore, by the monotonicity of $T_{n 1}$ on $\left(0, \frac{1}{2}\right)$ and $\left(\frac{1}{2}, 1\right)$, we know that there exist only two points $\beta_{2} \in\left(\alpha, \frac{1}{2}\right)$ and $\beta_{1} \in\left(\frac{1}{2}, 1\right)$ such that $T_{n 1}\left(\beta_{2}\right)=T_{n 1}\left(\beta_{1}\right)=\beta$. Set $f(x)=$ $T_{n 1}(x)-\beta_{2}$. Note that $f(\alpha)<0$ and $f\left(\beta_{2}\right)>0$. By the monotonicity of $f(x)$, we know that there exists a unique point $\beta_{3} \in\left(\alpha, \beta_{2}\right)$ such that $T_{n 1}\left(\beta_{3}\right)=\beta_{2}$. Proceeding like this, we can obtain a strictly monotone decreasing sequence $\left\{\beta_{m}\right\} \subset(\alpha, \beta)$, which satisfies $T_{n 1}\left(\beta_{m+1}\right)=\beta_{m}(m=2,3, \ldots)$. This implies $\#\left(J\left(T_{n 1}\right) \cap \mathbb{R}^{+}\right)=\infty$ since $\beta$ is a repelling fixed point.

(2) If $n>1$ is an odd number. By Lemma 3, we know that $T_{n 1}$ has only four fixed points $q_{1}<0, q_{2}=0,1$ and $q_{3}>1$. By a similar discussion as used in (3) in Theorem 1, we can deduce that there exist only two points $q_{1}^{*} \in\left(0, \frac{1}{2}\right)$ and $q_{3}^{*} \in\left(\frac{1}{2}, 1\right)$ such that $T_{n 1}\left(q_{1}^{*}\right)=q_{1}$ and $T_{n 1}\left(q_{3}^{*}\right)=q_{3}$. Furthermore, we can deduce that

$$
\begin{array}{ll}
\left(q_{1}, q_{1}^{*}\right) \subset A_{n 1}(0), & \left(q_{1}^{*}, q_{3}^{*}\right) \subset A_{\lambda}^{-1}(\infty), \\
\left(q_{3}, q_{3}\right) \subset A_{\lambda}(1), \quad\left(q_{3},+\infty\right) \subset A_{\lambda}(\infty) .
\end{array}
$$

Hence we get $J\left(T_{n 1}\right) \cap \mathbb{R}^{+}=\left\{q_{1}^{*}, q_{3}^{*}, q_{3}\right\}$, i.e., $\#\left(J\left(T_{n 1}\right) \cap \mathbb{R}^{+}\right)=3$.

(3) We know that $T_{n \gamma}$ has only one fixed point $q \in(1,+\infty)$. Obviously, $q$ is a repelling fixed point and $(q,+\infty) \subset A_{\lambda}(\infty)$. In what follows we distinguish two cases to discuss.

(a) If $n \geq 2$ and $\lambda=2 . T_{n 2}(z)=\left(\frac{z^{2}+1}{2 z}\right)^{n}, T_{n 2}$ has only six critical points $\pm 1, \pm i, 0, \infty$ and $T_{n 2}( \pm 1)=1, T_{n 2}( \pm i)=0, T_{n 2}(0)=\infty, T_{n 2}(\infty)=\infty$. Considering the real fixed points of $T_{n 2}, T_{n 2}(x)$ has no fixed point on $(-\infty, 0)$. Note that $T_{n \lambda}(x)$ is monotone decreasing on $(0,1)$ from $+\infty$ to 1 , then $T_{n \lambda}(x)$ has no fixed point on $(0,1)$.

Note that $T_{n \lambda}(x)$ is monotone decreasing on $(0,1)$ from $+\infty$ to 1 , then there exists a unique point $q_{1} \in(0,1)$ such that $T_{n 2}\left(q_{1}\right)=q$. By Lemma $5,\left[0, q_{1}\right) \subset D\left(-\frac{\lambda}{2}+1\right)$ and $\left(q_{1}, q\right) \subset$ $A_{\lambda}(1)$. We get $J\left(T_{n 2}\right) \cap \mathbb{R}^{+}=\left\{q_{1}, q\right\}$, i.e., $\#\left(J\left(T_{n 2}\right) \cap \mathbb{R}^{+}\right)=2$.

(b) If $n>2$ and $\lambda \in\left(2,2+\frac{1}{\sqrt[n-1]{2}-1}\right)$. By a similar discussion as (6) in Theorem 1 , we can easily deduce that $T_{n \lambda}^{2}(0)>T_{n \lambda}(0)$. Then $T_{n \lambda}(0)>1$. We conclude $T_{n \lambda}(0)>q$ since $T_{n \lambda}(x)<$ $x$ for $x \in(1, q)$. By $(2), T_{n \lambda}(x)$ is monotone decreasing on $(0,1)$. Then there exists a unique point $q_{1} \in(0,1)$ such that $T_{n 1}\left(q_{1}\right)=q$. Note that $q_{1} \in(0,1)$, then $J\left(T_{n \lambda}\right) \cap \mathbb{R}^{+}=\left\{q_{1}, q\right\}$, i.e., $\#\left(J\left(T_{n \lambda}\right) \cap \mathbb{R}^{+}\right)=2$.

(4) We also distinguish two cases to discuss. 
(c) If $n=2$ and $\lambda=3$. We know that $T_{23}$ has a unique repelling point $x_{0}=4$ on $(1,+\infty)$. It is easy to see that $T_{23}(x)<x$ for $x \in(1,4)$ and $T_{23}(x)>x$ for $x \in(4,+\infty)$. Note that $T_{23}(0)=4$ and $T_{23}(x)$ is monotone decreasing for $x \in(0,1)$, then $T_{23}(x) \in(1,4)$ for $x \in(0,1)$. Then $(0,4) \subset A_{\lambda}(1)$ and $(4,+\infty) \subset A_{\lambda}(\infty)$. We get $J\left(T_{23}\right) \cap \mathbb{R}^{+}=\left\{x_{0}\right\}$, i.e., $\#\left(J\left(T_{23}\right) \cap \mathbb{R}^{+}\right)=1$.

(d) If $n>2$ and $\lambda \in\left(2+\frac{1}{\sqrt[n-1]{2}-1},+\infty\right)$. By a similar discussion as (6) in Theorem 1, we also get $T_{n \lambda}^{2}(0)<T_{n \lambda}(0)$. Hence $T_{n \lambda}(0) \in(1, q)$ since $T_{n \lambda}(0)>1$. Note that $T_{n \lambda}(x)$ is monotone decreasing for $x \in[0,1)$, then $T_{n \lambda}(x) \in(1, q)$ for $x \in[0,1)$. This means $[0, q) \subset A_{\lambda}(1)$. We obtain $J\left(T_{n \lambda}\right) \cap \mathbb{R}^{+}=\{q\}$, i.e., $\#\left(J\left(T_{n \lambda}\right) \cap \mathbb{R}^{+}\right)=1$. The proof of Theorem 3 is completed.

\section{Competing interests}

The author declares that they have no competing interests.

\section{Author's contributions}

The author is entirely responsible for this research. The author read and approved the final manuscript.

\section{Acknowledgements}

The author would like to thank the referees for their valuable suggestions for improving this paper. This work was supported by the National Natural Science Foundation of China (No. 11371363, 11231009, 11261002, 11201474) and the Special Basic Scientific Research Funds of Central Universities in China.

Received: 4 April 2014 Accepted: 13 August 2014 Published: 11 Sep 2014

\section{References}

1. McMullen, C: Automorphisms of rational maps. In: Drasin, D (ed.) Holomorphic Functions and Moduli, pp. 1-31. Springer, New York (1988)

2. Eremenko, AE, Lyubich, MY: The dynamics of analytic transformations. Leningr. Math. J. 1, 563-634 (1990)

3. Beardon, AF: The components of a Julia set. Ann. Acad. Sci. Fenn., Ser. A 1 Math. 16(1), 173-180 (1991)

4. Morosawa, S: On the residual buried Julia sets of rational functions. Ergod. Theory Dyn. Syst. 17(1), $205-210$ (1997)

5. Qiao, J: The buried points on the Julia sets of rational and entire functions. Sci. China Ser. A 38, 1410-1419 (1995)

6. Qiao, J: Topological complexity of Julia sets. Sci. China Ser. A 40, 1158-1165 (1997)

7. Curry, CP, Mayer, JC: Buried point in Julia set. J. Differ. Equ. Appl. 5, 435-441 (2010)

8. Lee, TD, Yang, CN: Statistical theory of equations of state and phase transitions. II. Lattice gas and Ising model. Phys. Rev. 87, 410-419 (1952)

9. Yang, CN, Lee, TD: Statistical theory of equations of state and phase transitions. I. Theory of condensation. Phys. Rev. 87, 404-409 (1952)

10. Derrida, B, DeSeze, L, Itzykson, C: Fractal structure of zeros in hierarchical models. J. Stat. Phys. 33, 559-569 (1983)

11. Bleher, $\mathrm{P}$, Lyubich, M: Julia sets and complex singularities in hierarchical Ising models. Commun. Math. Phys. 141, 453-474 (1991)

12. Derrida, B, Itzykson, C, Luck, J: Oscillatory critical amplitudes in hierarchical models. Commun. Math. Phys. 94, 115-132 (1984)

13. Joao, C, Barata, A, Goldbaum, P: On the distribution and gap structure of Lee-Yang zeros for the Ising model, periodic and aperiodic couplings. J. Stat. Phys. 103, 857-891 (2001)

14. Qiao, J: Julia sets and complex singularities in diamond-like hierarchical Potts models. Sci. China Ser. A 48(3), 388-412 (2005)

15. Qiao, J, Li, Y: On connectivity of Julia sets of Yang-Lee zeros. Commun. Math. Phys. 222, 319-326 (2001)

16. Qiao, J, Gao, J: Jordan domain and Fatou set concerning diamond-like hierarchical models. Nonlinearity 40, 119-131 (2007)

17. Qiao, J, Yin, Y, Gao, J: Feigenbaum Julia sets of singularities of free energy. Ergod. Theory Dyn. Syst. 30, 1573-1591 (2010)

18. Beardon, A: Iteration of Rational Functions. Springer, Berlin (1991)

19. Carleson, L, Gamelin, TW: Complex Dynamics. Springer, Berlin (1991)

20. Milnor, J: Dynamics in One Complex Variable, 3rd edn. Annals of Math. Studies, vol. 160. Princeton University Press, Princeton (2006) 